\title{
Portación faríngea de Mycoplasma pneumoniae en niños chilenos
}

\author{
CAMILO PALMA S., M. ANGÉLICA MARTÍNEZ T., \\ MARCIA SALINAS S. y PAMELA ROJAS G.
}

\section{Asymptomatic pharyngeal carriage of Mycoplasma pneumoniae in Chilean children}

PCR has become a sensitive option for the rapid detection of Mycoplasma pneumoniae in respiratory specimens, but little is known of the frequency of its asymptomatic carriage or persistence in the throat after Mycoplasma disease in healthy children. To investigate the frequency of asymptomatic carriage of M. pneumoniae in children, we studied by PCR throat specimens from 185 respiratory asymptomatic children aged 1-14 years, enrolled at two pediatric ambulatory health services of Santiago, Chile from September 2002 through August 2003. M. pneumoniae DNA was detected in 4 $(2.16 \%)$ children. Positive specimens could represent either asymptomatic carriage or persistence of the organism from a previous disease.

Key words: Mycoplasma pneumoniae, Asymptomatic carriage, Children.

Palabras clave: Mycoplasma pneumoniae, Portación, Niños.

\section{Introducción}

Mycoplasma pneumoniae es uno de los agentes más frecuentes de neumonía y otras infecciones respiratorias agudas en niños y adultos jóvenes ${ }^{1}$. El diagnóstico oportuno de este microorganismo permite efectuar una terapia antimicrobiana específica y limitar su diseminación en la comunidad. La detección de IgM es el recurso de laboratorio más empleado actualmente en pediatría para el diagnóstico de neumonía por $M$. pneumoniae, pero su sensibilidad se aproxima solamente a $80 \%$, siendo dependiente de la dinámica de la respuesta inmune humoral y del momento de toma de la muestra de sangre ${ }^{2,5-7}$. En la infección por $M$. pneumoniae, la IgM aparece entre los 7 y 10 días de iniciado el cuadro clínico y rápidamente asciende, alcanzando un título máximo a los 10 a 30 días $^{6}$. Sin embargo, algunos pacientes no desarrollan IgM antes de las 2 semanas de iniciada la enfermedad, limitando la utilidad de este procedimiento diagnóstico ${ }^{6,7}$. Por otra parte, la especificidad de la detección de $\operatorname{IgM}$ mediante ELISA varía ampliamente entre 25 y $90 \%$, dependiendo del kit comercial utilizado ${ }^{3,4}$.

La amplificación del ADN mediante RPC ha sido señalada en algunos estudios como una alternativa sensible y específica para la detección de $M$. pneumoniae ${ }^{8-14}$. No obstante, se ha informado portación y persistencia de este microorganismo en la faringe luego de finalizado un cuadro respiratorio, lo que podría limitar la utilidad de esta técnica ${ }^{1-2,8-10,15}$. El objetivo de nuestro estudio es investigar la presencia de $M$. pneumoniae en muestras faríngeas de niños asintomáticos respiratorios.

Programa de Microbiología y Micología, Instituto de Ciencias Biomédicas, Facultad de Medicina, Universidad de Chile, Santiago, Chile (CPS, MAMT)

Servicio de Salud Metropolitano Central, Santiago, Chile. Ciclo de Formación y Destinación (MSS)

Medicina Familiar del Niño, Pontifica Universidad Católica de Chile, Santiago, Chile. Residente (PRG)

Recibido: 7 enero 2005

Aceptado: 22 junio 2005 


\section{Pacientes y Métodos}

Entre septiembre de 2002 y agosto de 2003 se obtuvo muestras de secreción faríngea en 185 niños, luego del consentimiento informado de sus padres. El promedio de edad fue 5,3 años (rango, 1 a 14 años); 98 eran niños y 87 niñas. Los niños fueron enrolados al estudio durante su atención en el programa de control de niño sano o en el Programa Ampliado de Inmunizaciones, en los consultorios de atención ambulatoria de Maipú y Villa O’Higgins de la Florida, Área Metropolitana, Santiago, Chile. Se excluyeron del estudio a niños con signos o síntomas de infección respiratoria o que hubieran recibido antimicrobianos en los últimos 30 días. Se tomó una muestra de secreción faríngea con tórula de algodón, la que fue inmediatamente depositada en $1,5 \mathrm{ml}$ de medio de transporte sacarosa fosfato (buffer 2SP). Las muestras fueron conservadas en refrigeración hasta su transporte en hielo al laboratorio.

Para el diagnóstico de $M$. pneumoniae mediante RPC se amplificaron 209 pb del gen de la adhesina P1, empleando los partidores descritos por Ieven y cols ${ }^{11}$. Para la extracción del ADN se centrifugó una alícuota de $0,4 \mathrm{ml}$ de la muestra clínica a 13.000 r.p.m. durante $30 \mathrm{~min}$. El pellet resultante fue suspendido en $100 \mathrm{ml}$ de buffer de lisis $\left(10 \mathrm{mM}\right.$ Tris- $\mathrm{HCl}, 50 \mathrm{mM} \mathrm{KCl}, 2 \mathrm{mM} \mathrm{MgCl}_{2}$, $0,1 \%$ Tritón $\mathrm{X}-100$ y $200 \mathrm{mg}$ por $\mathrm{ml}$ de proteinasa $\mathrm{K})$. La mezcla fue incubada a $60^{\circ} \mathrm{C}$ durante 60 min y luego a $94^{\circ} \mathrm{C}$ por 15 min y rápidamente enfriada. El ADN fue purificado por extracciones sucesivas con fenol cloroformo (fenol-cloroformo-alcohol isoamílico, 25:24:1) y cloroformo (cloroformo-alcohol isoamílico, 24:1). Las reacciones de amplificación fueron efectuadas en un volumen de $50 \mathrm{ml}$ conteniendo: buffer de amplificación $1 \mathrm{X}, 2.5 \mathrm{U}$ Taq polimerasa (Promega®), $200 \mathrm{mM}$ de cada nucleótido trifosfato, $2.5 \mathrm{mM}$ $\mathrm{MgCl}_{2}$ y $1 \mathrm{mM}$ de cada partidor. Los ciclos de amplificación consistieron en 4 min de denaturación a $94^{\circ} \mathrm{C}$, seguido por 40 ciclos de $1 \mathrm{~min}$ de denaturación a $94^{\circ} \mathrm{C}, 1 \mathrm{~min}$ de anillamiento a $65^{\circ} \mathrm{C}$ y 1 min de extensión a $72^{\circ} \mathrm{C}$, con un ciclo final de extensión de $5 \mathrm{~min}$ a $72^{\circ} \mathrm{C}$. Las amplificaciones fueron efectuadas en un termociclador MJ Research, Modelo MiniCycler®. Los productos de amplificación fueron separados en función de su tamaño, por electroforesis en geles de agarosa al $1,5 \%$, conteniendo $0,5 \mathrm{mg}$ por $\mathrm{ml} \mathrm{de}$ bromuro de etidio y posteriormente visualizados en transiluminador de luz ultravioleta. Como patrón de comparación del tamaño del ADN se utilizó ADN ladder de 100 pb. Como control positivo del proceso de amplificación se incluyó a la cepa FH de $M$. pneumoniae, la cual fue donada gentilmente por el Prof. Dr. Wolfgang Bredt (Alemania). Como control negativo se incluyó un tubo conteniendo todos los reactivos de la RPC excepto ADN, cada 8 muestras amplificadas.

\section{Resultados}

M. pneumoniae fue aislado de la faringe en 4 de los 185 (2,16\%) niños enrolados. En la Tabla 1 se muestra la frecuencia de detección del microorganismo según el estrato etario. М. pneumoniae fue detectado en 2/105 (1,9\%) niños de 1 a 4 años y en $2 / 80(2,5 \%)$ niños entre 5 y 14 años.

Tabla 1. Frecuencia de portación faríngea por Mycoplasma pneumoniae en 185 niños según estrato etario. Septiembre 2002-agosto 2003

\begin{tabular}{lcc}
\hline Edad (años) & $\begin{array}{c}\mathbf{N}^{\mathbf{o}} \text { niños } \\
\text { estudiados }\end{array}$ & $\begin{array}{c}\mathbf{N}^{\mathbf{0}}(\boldsymbol{\%}) \text { niños } \\
\text { colonizados }\end{array}$ \\
\hline $1-4$ & 105 & $2(1,9)$ \\
$5-14$ & 80 & $2(2,5)$ \\
Total & 185 & $4(2,16)$ \\
\hline
\end{tabular}

\section{Discusión}

Los resultados de nuestro estudio se suman a lo señalado en la literatura médica extranjera, demostrando la existencia de portación faríngea de $M$. pneumoniae en niños sanos. Foy documentó portación faríngea de $M$. pneumoniae en niños observando un aumento en su frecuencia durante los períodos de mayor incidencia del microorganismo ${ }^{1}$. Asimismo, Dorigo-Zetsma y cols demostraron $15 \%$ de portación en niños que estaban en contacto intrafamiliar con pacientes índices de infecciones respiratorias causadas por M. pneumoniae, durante una vigilancia centinela del virus influenza ${ }^{14}$. En un estudio anterior, caso control, los investigadores no encontraron portación de $M$. pneumoniae mediante RPC, pero solamente incluyeron muestras nasofaríngeas de 74 niños controles 5 .

También se ha demostrado portación de $M$. pneumoniae en adultos; Gnarpe y cols la demostraron en $4,6 \%$ de adultos sanos durante un período de baja incidencia del microorganismo y 
13,5\% de portación durante un período epidémi$\mathrm{co}^{15}$. Waring y cols comunicaron $15 \%$ de portación en un brote de infecciones causadas por M.pneumoniae que afectó a una comunidad religiosa cerrada en Nueva York ${ }^{16}$.

La portación faríngea de $M$. pneumoniae puede representar la persistencia de la bacteria luego de una infección sintomática. Foy documentó, en un estudio de observación longitudinal, la desaparición progresiva del microorganismo en el tiempo, alcanzando valores mínimos de detección a las 6 semanas post enfermedad ${ }^{1}$. La máxima persistencia del agente observada en el estudio fue de 4 meses ${ }^{1}$.

Kleemola y cols y Skakni y cols han sugerido que la carga de $M$. pneumoniae en la faringe es mayor en pacientes con neumonías de mayor gravedad, que en pacientes con neumonías de gravedad moderada o que en portadores del microorganismo, recomendando efectuar algún procedimiento cuantitativo o semicuantitativo de RPC para certificar el diagnóstico de enfermedad actual. Aunque ambos estudios incluyeron un bajo número de pacientes, sería importante confirmar estos hallazgos ${ }^{6,8}$.

La amplificación del ADN ha demostrado ser una técnica sensible para el diagnóstico de infección por $M$. pneumoniae en niños, pudiendo proporcionar los resultados dentro de 24 horas $^{5,8-12}$. Ha demostrado ser también útil para el diagnóstico de infecciones por $M$. pneumoniae en niños inmunocomprometidos y en lactantes bajo 12 meses de edad, en los cuales la respuesta inmune humoral a este microorganismo es menor ${ }^{8}$. La muestra de secreción faríngea es la más empleada para el diagnóstico de $M$. pneumoniae mediante RPC, porque tiene menor efecto inhibitorio sobre la reacción de amplificación del ADN que otras muestras respiratorias ${ }^{10}$. No obstante, debido a la existencia de portación y a la persistencia de $M$. pneumoniae post enfermedad, los resultados del diagnóstico de enfermedad mediante RPC de muestras obtenidas de sitios no estériles deben ser considerados probables, mientras que los resultados serológicos son de certeza.

Elegimos amplificar el gen que codifica la adhesina P1 porque está presente en varias copias en el genoma de $M$. pneumoniae, característica que podría otorgar mayor sensibilidad a la $\mathrm{RPC}^{11,17}$. Las reacciones de amplificación fueron efectuadas a partir de ADN purificado para evitar el efecto inhibitorio de las muestras en una RPC directa. No obstante, no utilizamos un control interno de amplificación y por ello no podemos descartar en nuestro estudio la existencia de mues- tras inhibitorias para RPC. Tjhie y cols demostraron $5,1 \%$ de muestras faríngeas inhibitorias en RPC directa versus $2 \%$ de muestras inhibitorias luego de la purificación del $\mathrm{ADN}^{10}$. La sensibilidad analítica de nuestra RPC es de 7,4 pg de ADN, levemente más sensible que la de AbeleHorn, que detecta $30 \mathrm{pg}$ de ADN, aproximadamente 19 UFC ó $1,9 \times 10^{3}$ bacterias y comparable a la sensibilidad de otras RPC simples ${ }^{12,18}$. Finalmente, se tomaron en consideración todas las medidas recomendadas para evitar contaminación de las muestras por arrastre de ADN; separación física de las áreas de trabajo, empleo de diferentes micropipetas en cada área, uso de puntas protegidas para la inoculación de los templados de ADN e inclusión de controles negativos de amplificación.

Recientemente se ha evaluado la especificidad de esta RPC con ADN de otros patógenos respiratorios y de bacterias respiratorias comensales demostrando amplificar el ADN de $M$. pneumoniae en forma específica ${ }^{18}$.

En conclusión, hemos demostrado que existe el estado de portación de $M$. pneumoniae en niños de la Región Metropolitana de Chile, siendo necesario aumentar el número de pacientes, como asimismo obtener muestras en diferentes períodos, para conocer la magnitud real de este fenómeno.

\section{Resumen}

La reacción de polimerasa en cadena (RPC) ha demostrado ser una alternativa sensible para el diagnóstico de enfermedad por $M$. pneumoniae proporcionando resultados en forma oportuna. Sin embargo, no se conoce bien la frecuencia de portación o la persistencia de este microorganismo en la faringe luego de una enfermedad respiratoria en niños, factores que podrían limitar la utilidad de este procedimiento de diagnóstico. Con el objeto de conocer la frecuencia de portación silenciosa de $M$. pneumoniae en niños, se estudiaron mediante RPC muestras faríngeas de 185 niños sin signos ni síntomas de infección respiratoria, enrolados en dos consultorios de la Región Metropolitana, Chile, entre septiembre de 2002 y agosto de 2003. M. pneumoniae fue detectado en $4(2,16 \%)$ niños. Las muestras positivas podrían representar portación o persistencia post enfermedad de $M$. pneumoniae.

\section{Referencias}

1.- Foy H M. Infections caused by Mycoplasma pneumoniae and possible carrier state in different populations of patients. Clin Infect Dis 1993; 17 (Suppl 1): S37-46. 
2.- Waris M E, Toikka P, Saarinen T, Nikkari S, Meurman O, Vainionpaa R, et al. Diagnosis of Mycoplasma pneumoniae pneumonia in children. J Clin Microbiol 1998; 36: 3155-9.

3.- Petitjean J, Vabret A, Gouarin S, Freymuth F. Evaluation of four commercial immunoglobulin $G$ (IgG)-and IgM-specific enzyme immunoassays for diagnosis of Mycoplasma pneumoniae infections. J Clin Microbiol 2002; 40: 165-71.

4.- Beersma M F, Dirven K, van Dam A P, Templeton K E, Claas E C, Goossens H. Evaluation of 12 commercial tests and the complement fixation test for Mycoplasma pneumoniae-specific immunoglobulin $\mathrm{G}$ (IgG) and IgM antibodies, with PCR used as the "gold standard". J Clin Microbiol 2005; 43: 2277-85

5.- Dorigo-Zetsma J W, Zaat S A, Wertheim-van Dillen $P$ M, Spanjaard L, Rijntjes J, van Waveren G, et al. Comparison of PCR, culture, and serological tests for diagnosis of Mycoplasma pneumoniae respiratory tract infections in children. J Clin Microbiol 1999; 37: 147.

6.- Kleemola S R, Karjalainen J E, Raty R K. Rapid diagnosis of Mycoplasma pneumoniae infection: clinical evaluation of a commercial probe test. J Infect Dis 1990; 162: 70-5.

7.- Moule J H, Caul E O, Wreghitt T G. The specific IgM response to Mycoplasma pneumoniae infection: interpretation and application to early diagnosis. Epidemiol Infect 1987; 99: 685-92.

8.- Skakni L, Sardet A, Just J, Landman-Parker J, Costil J, Moniot-Ville N, et al. Detection of Mycoplasma pneumoniae in clinical samples from pediatric patients by polymerase chain reaction. J Clin Microbiol 1992; 30: 2638-42.

9.- Williamson J, Marmion B P, Worswick D A, Kok T W, Tannock G, Herd R, et al. Laboratory diagnosis of Mycoplasma pneumoniae infection. Antigen capture and PCR-gene amplification for detection of the mycoplasma: problems and clinical correlation. Epidemiol Infect 1992; 109: 519-37.

10.- Tjhie J H, van Kuppeveld F J, Roosendaal R, Melchers W J, Gordijn R, MacLaren D M, et al. Direct
PCR enables detection of Mycoplasma pneumoniae in patients with respiratory tract infections. J Clin Microbiol 1994; 32: 11-6.

11.- Ieven M, Ursi D, Van Bever H, Quint W. Detection of Mycoplasma pneumoniae by two polymerase chain reactions and role of $M$. pneumoniae in acute respiratory tract infections in pediatric patients. J Infect Dis 1996 173: 1445-52.

12.- Abele-Horn M, Busch U, Nitschko H, Jacobs E, Bax R, Pfaff F, et al. Molecular approaches to diagnosis of pulmonary diseases due to Mycoplasma pneumoniae. J Clin Microbiol 1998; 36: 548-51.

13.- Tour M, Trabelsi A, Maatouk N, Hammami M. Amplification of P1 and 16S rRNA genes by nested PCR for detection of Mycoplasma pneumoniae in paediatric patients. Pathol Biol (Paris) 2005; 53: 914

14.- Dorigo-Zetsma J W, Wilbrink B, Van Der Nat H, Bartelds A I, Heijnen M L, Dankert J. Results of molecular detection of Mycoplasma pneumoniae among patients with acute respiratory infection and in their household contacts reveals children as human reservoirs. J Infect Dis 2001; 183: 675-8.

15.- Gnarpe J, Lundbäck A, Sundelöf B, Gnarpe H. Prevalence of Mycoplasma pneumoniae in subjectively healthy individuals. Scan J Infect Dis 1992; 24: 16164.

16.- Waring A L, Halse T A, Csiza C K, Carlyn C J, Arruda Musser K, Limberger R J. Development of a genomicsbased PCR assay for detection of Mycoplasma pneumoniae in a large outbreak in New York State. J Clin Microbiol 2001; 39: 1385-90.

17.- Himmelreich R H, Hilbert H, Plagens H, Pirkl E, Li B-L, Herrmann R. Complete sequence analysis of the genome of the bacterium Mycoplasma pneumoniae. Nucleic Acid Res 1996; 24: 4420-49.

18.- Pino Y, Salazar T. Análisis de la reacción en cadena de la polimerasa para la detección de Mycoplasma pneumoniae en adultos mayores con neumonía adquirida en la comunidad. 2004. Tesis conducente al grado de Licenciado en Kinesiología. Facultad de Medicina, Universidad de Chile.

Correspondencia a:

M. Angélica Martínez Tagle

E-mail: mamartin@med.uchile.cl 